\section{Prevalência de violência física por parceiro íntimo em homens e mulheres de Florianópolis, Santa Catarina, Brasil: estudo de base populacional}

\author{
Prevalence of intimate partner physical violence in \\ men and women from Florianópolis, Santa Catarina \\ State, Brazil: a population-based study
}

\author{
La prevalencia de la violencia física en parejas \\ íntimas entre hombres y mujeres de Florianópolis, \\ Santa Catarina, Brasil: estudio basado en la \\ población
}

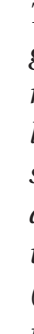
Prevalence of severe physical violence experienced by men only changed significantly according to marital status. Alcohol abuse by women increased the odds of suffering physical violence.

Domestic Violence; Spouse Abuse; Cross-Sectional Studies; Prevalence
Sheila Rubia Lindner 1 Elza Berger Salema Coelho 2 Carolina Carvalho Bolsoni 2 Paulo Fernando Rojas 2 Antonio Fernando Boing 2

\section{Resumo}

Investigou-se a associação entre sexo e violência física entre parceiros íntimos. Encontrou-se prevalência de sofrer qualquer violência física (17\%), violência física moderada $(16,6 \%)$ e violência física grave (7,3\%). Não houve diferença significativa para violência física moderada em homens e mulheres, porém, quanto mais grave o ato maior a ocorrência deste nas mulheres. Por meio de regressão logística testou-se a associação da violência com o sexo, ajustando-se às variáveis exploratórias. Mulheres de maior idade, viúvas/separadas, pobres, menos escolarizadas $e$ pretas registram maior probabilidade de sofrer violência. Nos homens, a prevalência de violência física grave apresentou alteração significativa apenas para estado civil. Uso abusivo de álcool por mulheres representou maior chance de sofrer violência física.

Violência Doméstica; Maus-Tratos Conjugais; Estudos Transversais; Prevalência 


\section{Introdução}

A violência praticada por parceiro íntimo é definida como "qualquer comportamento que cause mal físico, psicológico ou sexual, como atos de agressão física, abuso psicológico, comportamentos controladores, relações sexuais forçadas ou outras formas de coerção sexual" 1 (p. 91).

A violência praticada por parceiro íntimo é mundialmente reconhecida como um problema de saúde pública, sendo reportadas elevadas prevalências do fenômeno e graves consequências às suas vítimas ${ }^{2}$. Estudo conduzido pela Organização Mundial da Saúde (OMS) em dez países identificou expressivas prevalências de violência física (de $12,9 \%$ no Japão a $61 \%$ no Peru), violência sexual (de $6,2 \%$ no Japão a 58,6\% na Etiópia) e comportamento controlador (de $21 \%$ no Japão a 90\% na República da Tanzânia) perpetradas por parceiros íntimos contra mulheres 3 .

Ao analisar a violência praticada por parceiro íntimo segundo o sexo da vítima, estudos realizados no Canadá e nos Estados Unidos mostraram que as mulheres têm maior probabilidade do que os homens de serem machucadas e sofrerem formas mais graves de violência durante as agressões por parceiros íntimos (Instituto Brasileiro de Geografia e Estatística. Cidades@.http:/ / www.ibge.gov.br/cidadesat, acessado em 10/ Fev/2009) 4,5,6. Ao analisar dados sobre violência entre parceiros íntimos na população de 18 a 28 anos nos Estados Unidos, Whitaker et al. 7 descreveram que em $24 \%$ dos relacionamentos havia algum tipo de violência envolvida, e em metade destes casos os atos eram recíprocos entre homem e mulher. Nos casos de não reciprocidade, as mulheres foram as agressoras em cerca de $70 \%$ dos casos.

Na América Latina e no Caribe, aproximadamente uma entre três mulheres foi vítima de violência sexual, física ou psicológica durante sua vida ${ }^{8}$. No Brasil, um estudo de base populacional conduzido em 15 capitais brasileiras e no Distrito Federal reportou prevalência de agressão psicológica, abuso físico moderado e grave no casal de $78,3 \%, 21,5 \%$ e $12,9 \%$, respectivamente 9 .

O impacto da violência praticada por parceiro íntimo é apontado por Fanslow \& Robinson 10, que identificaram risco de suicídio três vezes maior entre as mulheres que sofreram violência física moderada e oito vezes maior entre as que sofreram violência física grave, quando comparadas com as que não sofreram violência física. Para os homens, o estudo conduzido por Coker et al. 2 nos Estados Unidos concluiu que a violência física ocasionada pelo parceiro íntimo representou a pior autopercepção de saúde, maiores taxas de sintomas depressivos, de uso abusivo de álcool e outras drogas, de doença mental crônica e de lesões, em comparação com os homens que não experimentaram este tipo abuso.

Apesar das severas consequências decorrentes da violência praticada por parceiro íntimo em ambos os sexos, a maioria das pesquisas que investigam a violência por parceiro íntimo é voltada às mulheres como vítimas e aos homens como agressores 11,12, e é majoritariamente realizada em países desenvolvidos 7,13,14,15,16,17. Portanto, há escassez de estudos que contemplem a violência por parceiro íntimo em homens ${ }^{11}$.

A maior parte dos trabalhos 9,18 tem investigado a violência no casal com base no depoimento da mulher, que relata a ocorrência da violência praticada por parceiro íntimo em ambos os sexos. Contudo, a violência conjugal não envolve apenas as mulheres, tornando-se necessário incorporar homens como participantes das pesquisas. Em consulta às bases bibliográficas PubMed, PsycINFO, LILACS e SciELO, com os termos Domestic Violence, Spouse Abuse, Battered Women, Epidemiology e Prevalence, foi encontrado apenas um estudo 19 brasileiro de base populacional cuja população-alvo era tanto homens quanto mulheres vítimas de violência. Assim, destaca-se a necessidade de se aprofundar no contexto brasileiro o conhecimento sobre a prevalência e as características da violência física cometida por parceiro íntimo tanto em homens quanto em mulheres.

O objetivo do presente estudo é investigar a associação entre sexo e violência física por parceiro íntimo em uma capital do Sul do Brasil.

\section{Métodos}

\section{Desenho e população do estudo}

Foi realizado estudo transversal de base populacional (EpiFloripa: http://www.epifloripa.ufsc. br). A coleta de dados ocorreu entre setembro de 2009 e janeiro de 2010 na zona urbana do Município de Florianópolis, capital do Estado de Santa Catarina. A população de referência foi composta por adultos de 20 a 59 anos de idade, residentes na área urbana do município, correspondendo a 249.530 pessoas $(61,1 \%$ do total da população) (Instituto Brasileiro de Geografia e Estatística. Censo Demográfico 2000. http://www.ibge.gov. br; acessado em 10/Fev/2009).

\section{Amostragem}

Para o cálculo do tamanho da amostra utilizaram-se como parâmetros prevalência de 50\%, nível de 95\% de confiança e erro amostral pre- 
visto de 3,5 pontos porcentuais. O valor obtido foi multiplicado por dois, considerando o efeito de delineamento por conglomerados, e foram acrescentados $10 \%$ para perdas previstas e $15 \%$ para estudos de associação, resultando em 1.979 pessoas. Como o presente trabalho fez parte de um amplo inquérito que analisou diversos desfechos em saúde, considerou-se a maior amostra calculada, equivalente a 2.016. Após coleta e análise dos dados foi possível calcular o poder da amostra entrevistada no presente estudo. Ela permitiu identificar razão de chances mínima de 1,48, adotando-se como parâmetros poder de $80 \%$, erro alfa de $5 \%$, razão de não expostos: expostos de 4:1 e prevalência nos não expostos de $16,1 \%$. O cálculo do tamanho da amostra foi realizado no programa Epi Info, versão 6.04 (Centers for Disease Control and Prevention, Atlanta, Estados Unidos).

O processo de seleção da amostra ocorreu por conglomerados em dois estágios. No primeiro, todos os 420 setores censitários urbanos da cidade foram dispostos em ordem crescente de acordo com a renda média mensal do chefe da família, e sorteou-se de maneira sistemática oitenta setores (oito em cada decil de renda). As unidades de segundo estágio foram os domicílios. Uma etapa de atualização do número de domicílios em cada setor foi necessária, uma vez que o censo mais recente, que provê a contagem de domicílios por setor, havia sido realizado em 2000. Para tanto, percorreram-se todos os setores censitários sorteados e realizou-se a contagem de domicílios particulares ocupados. O número de domicílios nos setores variou de 71 a 810 , correspondendo a um coeficiente de variação de 55\%. Para diminuí-lo, setores com menos de 150 domicílios foram agrupados, e com mais de 500 , divididos, respeitando-se o decil de renda e a proximidade geográfica. Assim, a amostra final contou com 63 setores, coeficiente de variação de $32 \%$ e 18 domicílios sorteados em cada uma dessas unidades geográficas.

\section{Coleta de dados}

Todos os moradores adultos dos domicílios selecionados eram elegíveis para entrevista e foram convidados a participar do estudo. Foram excluídos da pesquisa os indivíduos institucionalizados, com impedimento físico e/ou mental de responder ao questionário, e que se negaram a participar.

A coleta dos dados foi feita por intermédio de 35 entrevistadoras com o Ensino Médio completo. Utilizou-se um dispositivo eletrônico móvel (personal digital assistant - PDA) para o registro e armazenamento dos dados. Considerou-se perda o morador adulto, de domicílio sorteado, não encontrado após, no mínimo, quatro visitas, sendo obrigatoriamente uma no final de semana e outra no período noturno.

O controle de qualidade dos dados ocorreu semanalmente em 15\% das entrevistas, selecionadas por sorteio em cada setor. Informações-chave contidas no questionário foram verificadas em contato telefônico pelos supervisores de campo. A análise de concordâncias identificou valores de kappa variando de 0,6 a 1,0.

O presente trabalho estimou a prevalência de violência física por parceiro íntimo em mulheres e homens adultos em qualquer momento de suas vidas. Considerou-se parceiro íntimo todo companheiro ou ex-companheiro, independentemente da união formal, e namorados atuais ou anteriores, desde que mantivessem relações sexuais 20. Aplicou-se parte do questionário empregado pela OMS na pesquisa WHO Multicountry Study on Women's Health and Domestic Violence 21, validado no Brasil por Schraiber et al. 22 .

As questões relativas à violência física foram: (1) "se o parceiro já havia lhe dado um tapa ou jogado algo que poderia machucar"; (2) "se o parceiro havia empurrado ou dado um tranco/ chacoalhão"; (3) "se o parceiro machucou com um soco ou com algum objeto"; (4) "se deu algum chute, arrastou ou surrou o parceiro"; (5) "se o parceiro estrangulou ou queimou de propósito"; (6) "se o parceiro havia ameaçado usar ou realmente usou arma de fogo, faca ou outro tipo de arma contra o pesquisado".

Para identificar a ocorrência de qualquer violência física por parceiro íntimo era necessária resposta afirmativa a pelo menos uma das questões anteriores. Para ser considerada violência física moderada, o entrevistado precisava responder afirmativamente à questão 1 ou 2, e para classificar violência física como grave era necessário responder "sim" a uma das questões de 3 a 6 .

A violência fez parte do último bloco de perguntas da entrevista para que se estabelecesse uma relação de confiança entre o entrevistador e o entrevistado, e sempre com a presença apenas destas duas pessoas, em local reservado do domicílio. Sob nenhuma hipótese o cônjuge ouvia ou conhecia posteriormente a resposta de seu(sua) companheiro(a). Maiores detalhes metodológicos do estudo EpiFloripa estão disponíveis em Boing et al. 23 .

As variáveis demográficas e socioeconômicas obtidas foram sexo, idade em anos completos (posteriormente categorizada em 20 a 29, 30 a 39, 40 a 49 e 50 a 59), estado civil (casado ou com companheiro; solteiro; viúvo ou divorciado/separado), renda familiar per capita (estratificada em tercis), escolaridade em anos de estudos $(\geq 12$; 
$11-9 ; 8-5 ; \leq 4)$ e cor da pele autorreferida (branca, parda, preta, indígena e amarela; as duas últimas foram excluídas desta análise em razão do reduzido número de pessoas). A variável de comportamento relacionado à saúde foi o uso abusivo de álcool definido por meio do questionário Alcohol Use Disorders Identification Test (AUDIT) 24, composto por dez perguntas. As respostas a cada questão são pontuadas de 0 a 4 , sendo as maiores pontuações indicativas de problemas 24 . Sem uso abusivo de álcool, definiu-se pela pontuação igual ou inferior a 7 pontos, e com uso abusivo de álcool, considerado pela pontuação superior a 8 pontos $24,25,26$.

\section{Análise dos dados}

Inicialmente, foram calculadas as prevalências e os respectivos intervalos de $95 \%$ de confiança (IC95\%) de cada tipo de agressão isoladamente. Em seguida, estimaram-se as prevalências de violência física moderada, violência física grave, e ambas as violências físicas em cada categoria das covariáveis. Todas essas estimativas foram estratificadas por sexo.

Por fim, foi testada a associação de cada dimensão da violência com o sexo, ajustando-se pelas covariáveis. Para tal, realizou-se regressão logística bruta e ajustada, e obteve-se o odds ratio (OR) como medida de associação. Na análise, identificou-se interação entre as variáveis sexo e uso abusivo de álcool ( $\mathrm{p}<0,001)$. Dessa forma, foram criados os seguintes termos na análise de regressão: homem sem uso abusivo de álcool, mulher sem uso abusivo de álcool, homem com uso abusivo de álcool e mulher com uso abusivo de álcool. Foram incluídas na análise múltipla todas as variáveis que no modelo bivariado apresentaram $\mathrm{p}<0,20$. Na análise múltipla, considerou-se que o sexo foi associado com os desfechos quando o valor de $\mathrm{p}$ foi menor que 0,05 .

Todas as estimativas foram calculadas considerando-se o efeito de delineamento. As análises foram realizadas no programa Stata 9 (Stata Corp., College Station, Estados Unidos). A pesquisa foi aprovada pelo Comitê de Ética em Pesquisa com Seres Humanos da Universidade Federal de Santa Catarina (protocolo no 351/2008).

\section{Resultados}

Foram entrevistadas 1.720 pessoas, correspondendo a uma taxa de resposta de $85,3 \%$. Pouco mais da metade eram mulheres $(55,8 \%), 86 \%$ se referiram como sendo de cor/raça branca, $60,6 \%$ estavam casados ou vivendo com o companheiro, 42,9\% afirmaram ter 12 anos ou mais de estudos e a média de idades foi de 38,1 anos (DP $=1,12$ ano). Outras características da amostra estão descritas na Tabela 1.

As prevalências de ter sofrido qualquer violência física, violência física moderada e grave foram, respectivamente, de $17 \%, 16,6 \%$ e $7,3 \%$. Sofrer violência física apresentou prevalência de $16,1 \%$ para os homens e de $17,5 \%$ para as mulheres, sendo esta diferença estatisticamente significativa. Aproximadamente uma em cada seis mulheres e homens foi vítima de violência física moderada. Quando analisados separadamente os atos de violência, percebeu-se que tanto levar um tapa ou ser alvo de um objeto arremessado pelo companheiro quanto receber um tranco ou chacoalhão não diferiram estatisticamente entre os dois sexos, em que pese a medida pontual do segundo evento ser mais elevada entre as mulheres. Diferenças maiores foram observadas na prevalência de violência física grave $(8,5 \%$ das mulheres e 5,7\% dos homens), sendo que uma em cada 15 mulheres recebeu um soco ou foi machucada com algum objeto pelo companheiro, e entre os homens esta relação foi de um para 26. Para os demais atos de violência grave, as mulheres seguiram apresentando medida percentual maior que os homens, sendo maior a diferença quanto mais grave o ato (Tabela 2).

A Tabela 3 apresenta as prevalências de violência física moderada e grave segundo características demográficas e socioeconômicas. As prevalências de violência física e violência física grave entre os homens não apresentaram alteração significativa, sobretudo para idade, renda, escolaridade e raça. No entanto, verificou-se que homens viúvos/separados apresentaram prevalência três vezes maiores para estas violências em relação aos homens casados. Também entre as mulheres foram observadas variações nas prevalências de violência segundo as características demográficas e socioeconômicas. O fenômeno foi mais comum entre as mulheres com maior idade, as viúvas ou separadas, as mais pobres, menos escolarizadas e pretas. No entanto, verificou-se que mulheres com menos de cinco anos de estudos apresentaram prevalência três vezes maiores de violência moderada e cinco vezes maiores para a violência grave em comparação com aquelas com doze ou mais anos de estudos.

As Figuras 1, 2 e 3 apresentam a prevalência de violência física em homens e mulheres segundo o uso abusivo de álcool. Verificou-se que as mulheres, fazendo ou não uso abusivo do álcool, apresentaram maior prevalência de sofrer violência física, independentemente da gravidade da violência (Figuras 2 e 3). Para a violência física grave (Figura 3), a diferença da prevalência entre 
Características sociodemográficas da amostra. Florianópolis, Santa Catarina, Brasil, 2009.

\begin{tabular}{|c|c|c|}
\hline Características sociodemográficas & $n(\%)$ & IC95\% \\
\hline \multicolumn{3}{|l|}{$\operatorname{Sexo}(n=1.720)$} \\
\hline Masculino & $761(44,2)$ & 42,$3 ; 46,6$ \\
\hline Feminino & $959(55,8)$ & 53,$4 ; 57,7$ \\
\hline \multicolumn{3}{|l|}{ Idade (anos) $(n=1.720)$} \\
\hline $20-29$ & $540(31,4)$ & 28,$1 ; 37,8$ \\
\hline $30-39$ & $392(22,8)$ & 20,$3 ; 25,5$ \\
\hline $40-49$ & $438(25,5)$ & 21,$8 ; 28,1$ \\
\hline $50-59$ & $350(20,3)$ & 16,$9 ; 21,9$ \\
\hline \multicolumn{3}{|l|}{ Estado civil $(n=1.720)$} \\
\hline Casado ou com companheiro & $1.043(60,6)$ & 56,$5 ; 63,6$ \\
\hline Solteiro & $503(29,2)$ & 26,$3 ; 33,5$ \\
\hline Viúvo ou divorciado/separado & $174(10,1)$ & 8,$2 ; 11,8$ \\
\hline \multicolumn{3}{|l|}{ Renda per capita $(\mathrm{n}=1.685)$} \\
\hline Tercil 1 (menor) & $564(33,5)$ & 26,$1 ; 39,0$ \\
\hline Tercil 2 & $562(33,3)$ & 29,$6 ; 36,8$ \\
\hline Tercil 3 & $559(33,2)$ & 27,$8 ; 40,4$ \\
\hline \multicolumn{3}{|c|}{ Escolaridade (anos de estudo) $(n=1.716)$} \\
\hline$\geq 12$ & $737(42,9)$ & 36,$9 ; 50,8$ \\
\hline $11-9$ & $568(33,1)$ & 28,$8 ; 38,0$ \\
\hline $8-5$ & $253(14,7)$ & 11,$2 ; 16,7$ \\
\hline$\leq 4$ & $158(9,2)$ & 6,$4 ; 11,1$ \\
\hline \multicolumn{3}{|l|}{ Cor/Raça $(n=1.678)$} \\
\hline Branca & $1.444(86)$ & 82,$3 ; 89,3$ \\
\hline Parda & $147(8,7)$ & 6,$7 ; 11,5$ \\
\hline Preta & $87(5,2)$ & 3,$3 ; 6,9$ \\
\hline \multicolumn{3}{|l|}{ Uso abusivo de álcool $(n=1.720)$} \\
\hline Não & $1.403(81,5)$ & 78,$5 ; 84,5$ \\
\hline Sim & $307(18,5)$ & 15,$6 ; 21,5$ \\
\hline
\end{tabular}

IC95\%: intervalo de 95\% de confiança.

homens e mulheres que fazem uso abusivo do álcool é sensivelmente maior (12,5\%). A diferença da prevalência entre as mulheres que fazem ou não uso abusivo do álcool também é maior para a violência física grave, chegando a ser 12,8\% maior para aquelas que fazem uso abusivo de álcool. Para os demais tipos de violência física, essa diferença não ultrapassa os 3\% (Figura 3).

Quando foram testados os desfechos segundo os termos de interação de consumo de álcool e sexo (Tabela 4), observou-se na análise ajustada que as mulheres que reportaram uso abusivo de álcool apresentaram maior chance de ter sofrido violência física, sendo que o OR chegou a 4,18 no caso de violência física grave e a 2,50 na violência física moderada, em comparação com os homens que não fazem uso abusivo de álcool. Também houve diferença estatisticamente signi- ficativa da categoria de referência com as mulheres que não fazem uso abusivo de álcool no caso da violência física moderada e qualquer tipo de violência sofrida.

\section{Discussão}

Os resultados do presente trabalho demonstram que a prevalência de violência física moderada em homens e mulheres é semelhante, porém, quando é analisada segundo variáveis demográficas e socioeconômicas, as mulheres apresentam maior vulnerabilidade, principalmente em relação à menor escolaridade e renda. Essa diferença fica mais evidente quando é analisada em relação à violência física grave, assim como ao uso abusivo do álcool, que representou um 
Distribuição das respostas quanto à violência física em homens e mulheres. Florianópolis, Santa Catarina, Brasil, 2009.

\begin{tabular}{|c|c|c|c|}
\hline \multirow[t]{2}{*}{ Questões } & \multirow[t]{2}{*}{ n (\%) } & \multicolumn{2}{|c|}{ Sexo } \\
\hline & & Homem (IC95\%) & Mulher (IC95\%) \\
\hline Violência física moderada & $285(16,6)$ & $15,8(13,0 ; 18,6)$ & $17,0(14,3 ; 19,6)$ \\
\hline $\begin{array}{l}\text { Deu-lhe um tapa ou jogou algo no(a) Sr.(a) que } \\
\text { poderia machucá-lo(a)? }\end{array}$ & $209(12,2)$ & $12,2(9,5 ; 14,9)$ & $11,9(9,3 ; 14,4)$ \\
\hline Empurrou-o(a) ou deu-lhe um tranco/chacoalhão? & $233(23,6)$ & $11,1(8,7 ; 13,4)$ & $15,1(12,8 ; 17,3)$ \\
\hline Violência física grave & $125(7,3)$ & $5,7(3,8 ; 7,6)$ & $8,5(6,5 ; 10,5)$ \\
\hline $\begin{array}{l}\text { Machucou-o(a) com um soco ou com algum } \\
\text { objeto? }\end{array}$ & $94(5,5)$ & $3,8(2,3 ; 5,4)$ & $6,5(4,7 ; 8,4)$ \\
\hline Deu-Ihe um chute, arrastou ou surrou o(a) Sr.(a)? & $73(4,3)$ & $2,6(1,3 ; 4,0)$ & $5,5(3,9 ; 7,0)$ \\
\hline Estrangulou ou queimou o(a) Sr.(a) de propósito? & $26(1,5)$ & $0,6(0,06 ; 1,2)$ & $2,2(1,3 ; 3,1)$ \\
\hline $\begin{array}{l}\text { Ameaçou usar ou realmente usou arma de fogo, } \\
\text { faca ou outro tipo de arma contra o(a) Sr.(a)? }\end{array}$ & $47(2,7)$ & $1,5(0,4 ; 2,6)$ & $4,0(2,5 ; 5,5)$ \\
\hline Qualquer violência física & $293(17,0)$ & $16,1(13,3 ; 19,0)$ & $17,5(15,0 ; 20,2)$ \\
\hline
\end{tabular}

IC95\%: intervalo de 95\% de confiança.

importante fator de associação à violência física grave em mulheres.

Este estudo transversal permitiu apresentar a prevalência da violência tanto em homens quanto em mulheres. Vale destacar alguns aspectos positivos relacionados às questões metodológicas, como a alta taxa de resposta, que foi semelhante em todos os estratos de renda dos setores censitários, e a composição por faixa etária, sendo os valores obtidos no presente trabalho semelhantes aos das estimativas do Instituto Brasileiro de Geografia e Estatística (IBGE) para a população adulta de Florianópolis em 2009. As perdas apresentaram igualmente distribuição uniforme nos decis de renda familiar, contribuindo para a inferência da amostra.

Limitações dos estudos transversais precisam ser consideradas, principalmente o viés de informação e o viés recordatório, condicionados ao autorrelato da ocorrência de episódios de violência física por parceiro íntimo. Algumas formas de violência dependem da boa capacidade de memória do entrevistado e ainda de que a violência seja marcante, não seja esquecida com facilidade. Situações consideradas mais corriqueiras como um empurrão ou um tapa recebido pelo homem que não geram nenhuma consequência podem não ser lembradas. Ainda assim, as mulheres tendem mais a lembrar de situações de violência, pela maior repercussão física e psicológica que elas podem ocasionar 21,27. Um último aspecto metodológico relevante e que deve ser mencionado é o período de tempo em que a violência praticada por parceiro íntimo foi investigada. No presente trabalho analisou-se a ocorrência de violência ao longo da vida, o que pode maximizar as estimativas de prevalências observadas quando comparamse tais resultados com estudos que restringem a experiência a períodos como 6 ou 12 meses, por exemplo.

Como já mencionado por Anacleto et al. 18, os estudos de base populacional parecem detectar melhor os casos de violência física moderada, o que, de certa forma, tornaria maior a violência perpetrada pela mulher em relação à do homem. Porém é importante destacar que de maneira geral a mulher não perpetra agressões contra o parceiro na mesma intensidade e severidade que é cometida contra ela pelo parceiro homem 28 . Alguns estudos mostraram que as mulheres seriam mais prováveis a usar a agressão física do que os homens 29,30 , que tenderiam a usar armas e outros objetos como formas de agressão que não precisam da força física, além do uso da violência psicológica 31 que é um tipo de violência predominantemente cometida por mulheres contra seus parceiros.

As prevalências encontradas nas mulheres foram em geral mais baixas do que a relatada por mulheres em estudos populacionais que usaram o mesmo instrumento apenas com mulheres no Brasil 20,26,32. Esse achado pode ser explicado uma vez que neste estudo considerou-se outros desfechos de saúde, e a literatura aponta uma maior fragilidade de revelação em estudos deste tipo do que nos dedicados exclusivamente à violência 10,13 . 
Prevalência de sofrer violência física moderada e violência física grave em homens e mulheres, segundo variáveis demográficas e socioeconômicas. Florianópolis, Santa Catarina, Brasil, 2009.

\begin{tabular}{|c|c|c|c|c|}
\hline & \multicolumn{2}{|c|}{ Homens } & \multicolumn{2}{|c|}{ Mulheres } \\
\hline & $\begin{array}{c}\text { Violência física } \\
\text { moderada }\end{array}$ & $\begin{array}{l}\text { Violência física } \\
\text { grave } \\
\text { Prevalência }\end{array}$ & $\begin{array}{l}\text { Violência física } \\
\text { moderada } \\
95 \%)]\end{array}$ & $\begin{array}{c}\text { Violência física } \\
\text { grave }\end{array}$ \\
\hline \multicolumn{5}{|l|}{ Idade (anos) } \\
\hline $20-29$ & $18,7(13,2 ; 24,3)$ & $4,0(1,4 ; 6,6)$ & $11,8(8,2 ; 15,4)$ & $5,0(2,3 ; 7,9)$ \\
\hline $30-39$ & $17,8(12,1 ; 23,5)$ & $6,2(1,5 ; 11,0)$ & $16,3(11,2 ; 21,4)$ & $7,0(3,8 ; 10,0)$ \\
\hline $40-49$ & $14,7(9,8 ; 19,6)$ & $8,0(3,8 ; 12,2)$ & $21,3(15,4 ; 27,2)$ & $11,4(6,6 ; 16,2)$ \\
\hline $50-59$ & $9,4(3,3 ; 15,5)$ & $5,5(0,5 ; 10,7)$ & $20,3(14,3 ; 26,3)$ & $12,0(6,8 ; 17,1)$ \\
\hline \multicolumn{5}{|l|}{ Estado civil } \\
\hline Solteiro & $16,7(11,6 ; 21,8)$ & $3,8(1,5 ; 6,0)$ & $13,6(9,0 ; 18,4)$ & $6,5(3,5 ; 9,4)$ \\
\hline $\begin{array}{l}\text { Casado/Com } \\
\text { companheiro }\end{array}$ & $13,6(10,3 ; 16,8)$ & $5,6(3,4 ; 8,0)$ & $14,0(11,0 ; 17,0)$ & $6,1(4,1 ; 8,1)$ \\
\hline Viúvo/Separado & $32,1(16,0 ; 48,2)$ & $16,0(3,4 ; 28,5)$ & $38,6(29,7 ; 47,5)$ & $24,3(16,3 ; 32,3)$ \\
\hline \multicolumn{5}{|l|}{ Renda per capita } \\
\hline Tercil 1 (menor) & $14,4(9,5 ; 19,3)$ & $7,0(2,8 ; 11,2)$ & $23,0(19,3 ; 26,7)$ & $13,0(9,5 ; 16,4)$ \\
\hline Tercil 2 & $15,2(10,8 ; 19,7)$ & $5,8(2,4 ; 9,1)$ & $15,1(10,8 ; 19,3)$ & $7,3(4,5 ; 10,1)$ \\
\hline Tercil 3 & $18,0(12,5 ; 23,6)$ & $5,0(2,0 ; 7,8)$ & $12,3(8,1 ; 16,5)$ & $5,1(2,0 ; 8,3)$ \\
\hline \multicolumn{5}{|l|}{$\begin{array}{l}\text { Escolaridade (anos } \\
\text { de estudo) }\end{array}$} \\
\hline$\leq 4$ & $9,3(2,5 ; 16,0)$ & $4,8(-0,6 ; 10,3)$ & $32,4(19,2 ; 45,5)$ & $22,3(12,3 ; 32,3)$ \\
\hline $5-8$ & $16,6(10,3 ; 22,9)$ & $5,5(1,1 ; 9,9)$ & $21,2(13,4 ; 29,0)$ & $11,9(6,1 ; 17,6)$ \\
\hline $9-11$ & $16,1(11,5 ; 20,7)$ & $5,9(2,6 ; 9,1)$ & $18,7(13,5 ; 24,0)$ & $9,0(4,6 ; 13,5)$ \\
\hline$\geq 12$ & $17,0(12,4 ; 21,4)$ & $6,0(3,1 ; 8,7)$ & $11,4(8,5 ; 14,4)$ & $4,4(2,5 ; 6,2)$ \\
\hline \multicolumn{5}{|l|}{ Cor/Raça } \\
\hline Branca & $15,6(12,2 ; 18,9)$ & $5,4(3,4 ; 7,4)$ & $15,5(13,0 ; 18,1)$ & $7,8(5,6 ; 10,0)$ \\
\hline Parda & $20,5(11,5 ; 29,6)$ & $9,5(2,0 ; 17,0)$ & $19,9(9,7 ; 30,0)$ & $9,7(3,0 ; 16,5)$ \\
\hline Preta & $8,9(-1,5 ; 19,3)$ & $4,7(-1,6 ; 11,0)$ & $28,3(14,2 ; 42,4)$ & $16,8(4,3 ; 29,2)$ \\
\hline
\end{tabular}

IC95\%: intervalo de $95 \%$ de confiança.

Nas análises estratificadas por sexo, o presente trabalho demonstrou que as mulheres declararam cometer tantos atos de violência moderada quanto os homens. Esse achado é corroborado por estudos 8,33 que indicaram prevalência similar ou maior de violência perpetrada por mulheres contra o parceiro. As prevalências de violência praticada por parceiro íntimo encontradas são mais baixas do que as de algumas pesquisas de populações específicas dos Estados Unidos e em um estudo recente de população urbana no Brasil 9,11,13,34, "empurrar, agarrar ou sacudir" e "bater com alguma coisa" foram as formas mais prevalentes de violência praticada por parceiro íntimo entre os atos leves e os atos graves de violência. Talvez isso resulte do fato de os ho- mens subnotificarem a vitimização da violência mais do que as mulheres, ou o contrário, que as mulheres tendem a falar mais sobre a violência - tanto as que cometem quanto as que sofrem 11,15,19. Estudos revelam que as mulheres, mesmo quando iniciam um episódio de violência praticada por parceiro íntimo, o fazem com maior frequência em situações de autodefesa, enquanto os homens utilizam a violência com o objetivo de intimidar a parceira e mostrar autoridade. Mesmo assim, quando isso ocorre, considera-se a mulher como perpretadora ou envolvida em um ato de agressão mútua, pois o questionário utilizado não faz avaliação dos fatores desencadeadores da violência 27 . 
Figura 1

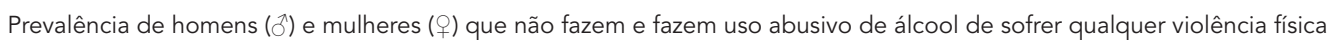
Florianópolis, Santa Catarina, Brasil, 2009.

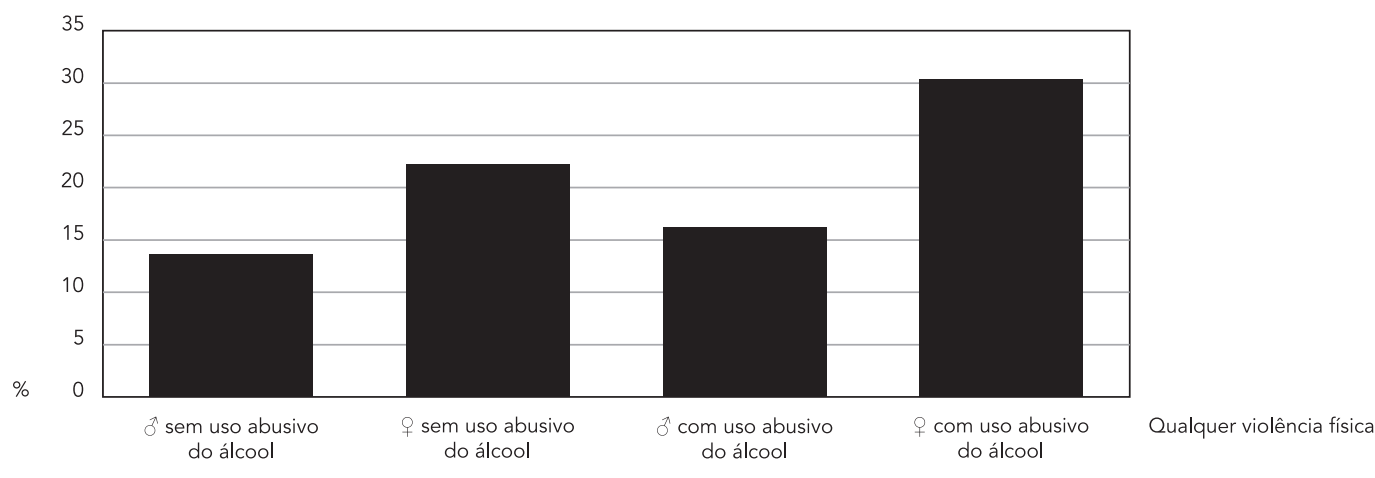

Figura 2

Prevalência de homens $\left({ }^{(}\right)$e mulheres ( $(+)$que não fazem e fazem uso abusivo de álcool de sofrer violência física moderada. Florianópolis, Santa Catarina, Brasil, 2009.

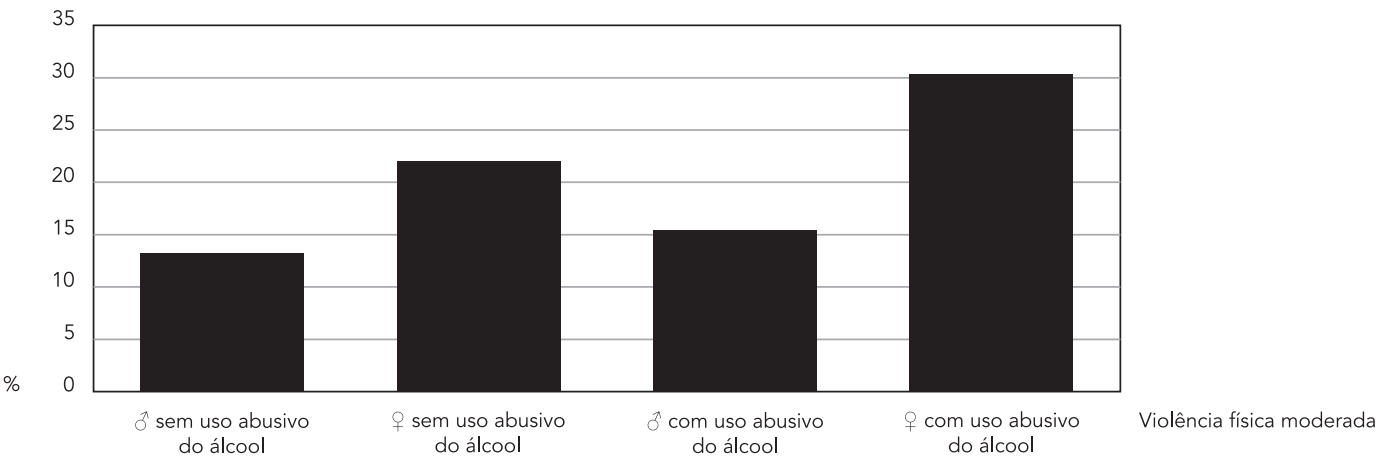

Nesse sentido, para o melhor entendimento dos atos de violência, é preciso considerar a dinâmica dessas relações e o contexto familiar, que agem em conjunto com os demais fatores individuais e estruturais envolvidos na violência 35 .

Os achados desta pesquisa corroboram os de outros estudos 36 no que se refere à maior prevalência de violência moderada e grave nas mulheres com baixa escolaridade. No estudo das 15 capitais brasileiras e Distrito Federal ${ }^{9}$ a taxa da prevalência para mulheres com Ensino Fundamental completo é de $18,9 \%$, e para aquelas que têm menos anos de estudos esta taxa sobe para $31 \%$. Para Anacleto et al. 18, a escolaridade também apresenta associação com a violência física grave ( $\mathrm{p}<0,001)$, sendo que mulheres com mais de 12 anos de estudos apresentaram as menores taxas $(4,8 \%)$.

Nesta pesquisa, pessoas que fazem uso abusivo de álcool e que sofreram violência física grave independentemente do sexo, da idade, da renda e da escolaridade continuaram a apresentar maior chance para o referido desfecho. Segundo Nagassar et al. 37, o abuso do álcool e de outras 
Figura 3

Prevalência de homens ( $\left({ }^{\wedge}\right)$ e mulheres (\$) que não fazem e fazem uso abusivo de álcool de sofrer violência física grave.

Florianópolis, Santa Catarina, Brasil, 2009.

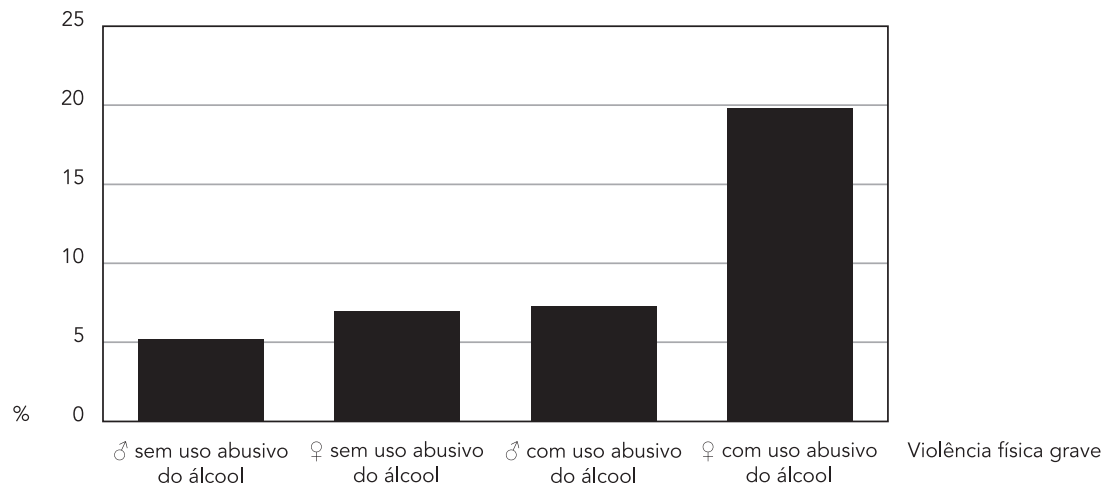

Tabela 4

Odds ratio bruta e ajustada de homens e mulheres sem ou com uso abusivo de álcool sofrerem qualquer violência física, violência física moderada ou violência física grave e variáveis demográficas e socioeconômicas. Florianópolis, Santa Catarina, Brasil, 2009

\begin{tabular}{|c|c|c|c|c|}
\hline & $\begin{array}{l}\text { Homem sem uso } \\
\text { abusivo de álcool }\end{array}$ & $\begin{array}{l}\text { Mulher sem uso } \\
\text { abusivo de álcool }\end{array}$ & $\begin{array}{l}\text { Homem com uso } \\
\text { abusivo de álcool }\end{array}$ & $\begin{array}{l}\text { Mulher com uso } \\
\text { abusivo de álcool }\end{array}$ \\
\hline \multicolumn{5}{|l|}{ Qualquer violência física } \\
\hline OR bruta (IC95\%) & 1,00 & $1,82(1,26 ; 2,63)$ & $1,22(0,92 ; 1,62)$ & $2,77(1,66 ; 4,63)$ \\
\hline Valor de $\mathrm{p}$ & & 0,002 & 0,145 & $<0,001$ \\
\hline OR ajustada * (IC95\%) & 1,00 & $1,64(1,12 ; 2,40)$ & $1,10(0,82 ; 1,47)$ & $2,42(1,30 ; 4,51)$ \\
\hline Valor de $p$ & & 0,012 & 0,510 & 0,006 \\
\hline \multicolumn{5}{|l|}{ Violência física moderada } \\
\hline OR bruta (IC95\%) & 1,00 & $1,85(1,27 ; 2,70)$ & $1,21(0,90 ; 1,62)$ & $1,21(0,90 ; 1,62)$ \\
\hline Valor de $\mathrm{p}$ & & 0,002 & 0,198 & $<0,001$ \\
\hline OR ajustada * (IC95\%) & 1,00 & $1,66(1,12 ; 2,43)$ & $1,08(0,80 ; 1,46)$ & $2,50(1,33 ; 4,65)$ \\
\hline Valor de $p$ & & 0,012 & 0,624 & 0,005 \\
\hline \multicolumn{5}{|l|}{ Violência física grave } \\
\hline OR bruta (IC95\%) & 1,00 & $1,37(0,66 ; 2,85)$ & $1,44(0,86 ; 2,41)$ & $4,52(2,37 ; 8,60)$ \\
\hline Valor de $\mathrm{p}$ & & 0,395 & 0,165 & $<0,001$ \\
\hline OR ajustada * (IC95\%) & 1,00 & $1,26(0,60 ; 2,66)$ & $1,26(0,73 ; 2,18)$ & $4,18(1,93 ; 9,07)$ \\
\hline Valor de $\mathrm{p}$ & & 0,527 & 0,389 & $<0,001$ \\
\hline
\end{tabular}

IC95\%: intervalo de 95\% de confiança.

* Ajustada por idade, raça, estado civil, renda e escolaridade.

drogas foi considerado como um dos principais motivos para a violência física (37\%), além de ser uma das principais associações para a violência física pelas mulheres vitimizadas $(\mathrm{OR}=6,02) 38$. Zaleski et al. 19, que investigaram a violência pra- ticada por parceiro íntimo e o consumo de álcool, apontam que de cada dez homens e mulheres, quatro e um, respectivamente, relataram a ingestão de bebida alcoólica durante os episódios de violência, sendo que os homens relataram índi- 
ces semelhantes de consumo de bebida alcoólica no caso de violência recíproca e durante atos de violência em que eles foram os autores. Porém, quando questionados sobre o uso de álcool durante o episódio de violência pelo parceiro, quase a metade das mulheres e um terço dos homens relataram este acontecimento.

Além do consumo de álcool ser considerado fator cuja presença tende a aumentar as chances de ocorrência de atos violentos $32,39,40$, é possível supor que a ingestão de bebidas alcoólicas pode ser estratégia adotada pelas vítimas para lidar com o estresse provocado pelo contexto de violência 32,41 .

O consumo excessivo de álcool é uma das mais controversas variáveis estudadas como causadoras da violência. Evidências de pesquisa indicam que o beber intenso contribui para a violência, mas isto não quer dizer que o álcool é condição primária, necessária e suficiente para a violência. Nesse sentido, o álcool não determinaria tais condutas, mas contribuiria para que se manifestassem de maneira mais intensa ou severa ${ }^{42}$. Para Sullivan et al. ${ }^{43}$, a gravidade da violência por parceiro íntimo é um dos fatores de risco para o consumo de álcool por mulheres expostas a este tipo de violência.

Existem poucas evidências que permitam concluir acerca da existência de uma relação de causalidade entre abuso de substâncias e violência doméstica 32 . Entretanto, Bennet \& O’Brien 44 destacam que parece haver uma bidirecionalidade entre essas duas variáveis, sendo que um problema parece aumentar o risco do outro. Dessa forma, defendem que serviços voltados ao atendimento de vítimas e agressores, bem como aqueles que estão focados nos usuários, investiguem essa co-ocorrência e trabalhem em regime de colaboração.

Os resultados obtidos neste estudo afirmam a importância de estudar a violência entre parceiros íntimos, demonstrando a bidirecionalidade deste fenômeno, apontando o homem não só como autor da violência, mas configurando-o como o que sofre. Fatores que permeiam essas relações são importantes de serem evidenciados e divulgados, para que se traduzam em implantação de políticas públicas voltadas a homens e mulheres em situação de violência por parceiro íntimo.

\section{Resumen}

Se investigó la asociación entre el sexo y la violencia física entre parejas íntimas. La prevalencia de sufrir violencia física (17\%), la violencia física moderada (16,6\%) y la violencia física grave $(7,3 \%)$. No hubo diferencia significativa en la violencia física moderada en hombres y mujeres, sin embargo, más grave es la mayor ocurrencia de este hecho en las mujeres. Por regresión logística se evaluó la relación entre la violencia y el se$x o, y$ el ajuste de las variables exploratorias. Las mujeres mayores de edad, viudas/separadas, más pobres, menos educadas y negras son más propensas a sufrir violencia. En los hombres, la prevalencia de la violencia física grave cambió significativamente sólo con el estado civil. El abuso de alcohol por mujeres representan un mayor riesgo de sufrir violencia.

Violencia Doméstica; Maltrato Conyugal; Estudios Transversales; Prevalencia

\section{Colaboradores}

S. R. Lindner participou da concepção do projeto, da análise e interpretação dos dados e da redação do artigo. E. B. S. Coelho orientou o projeto, a análise e a interpretação dos dados, realizou a revisão crítica do conteúdo e deu aprovação final para publicação. C. C. Bolsoni e P. F. Rojas colaboraram na pesquisa bibliográfica e da redação do texto. A. F. Boing coordenou a pesquisa de campo e realizou a revisão crítica do conteúdo.

\section{Agradecimentos}

Agradecemos à Dra. Nilza Nunes da Silva, do Departamento de Epidemiologia da Faculdade de Saúde Pública, Universidade de São Paulo, pelo auxílio nos procedimentos da amostra; aos técnicos do IBGE e da Secretaria Municipal de Saúde de Florianópolis pelo auxílio na operacionalização deste estudo; ao CNPq (processo ${ }^{\circ}$ 485327/2007-4) pelo financiamento. 
1. Krug EG, Dahlberg LL, Mercy JA, Zwi AB, Lozano R Relatório mundial sobre violência e saúde. Genebra: Organização Mundial da Saúde; 2002.

2. Coker AL, Davis KE, Arias I, Desai S, Sanderson M, Brandt HM, et al. Physical and mental health effects of intimate partner violence for men and women. Am J Prev Med 2002; 23:260-8.

3. Garcia-Moreno C, Jansen HAFM, Ellsberg M, Heise $\mathrm{L}$, Watts $\mathrm{CH}$. Prevalence of intimate partner violence: findings from the WHO multi-country study on women's health and domestic violence. Lancet 2006; 368:1260-9.

4. Heise L. Gender-based abuse: the global epidemic. Cad Saúde Pública 1994; 10 Suppl 1:135-45.

5. Walby S, Allen J. Domestic violence, sexual assault and stalking: findings from the British Crime Survey. London: Home Office Research, Development and Statistics Directorate; 2004. (Home Office Research Study, 276).

6. Hasselmann MH, Reichenheim ME. Adaptação transcultural da versão em português da Conflict Tactics Scales Form R (CTS-1), usada para aferir violência no casal: equivalências semântica e de mensuração. Cad Saúde Pública 2003; 19:1083-93.

7. Whitaker DJ, Haileyesus T, Swahn M, Saltzman LS. Differences in frequency of violence and reported injury between relationships with reciprocal and nonreciprocal intimate partner violence. Am J Public Health 2007; 97:941-7.

8. Pan American Health Organization. Health in the Americas 2007. Volume I - regional. Washington DC: Pan American Health Organization; 2007.

9. Reichenheim ME, Moraes CL, Szklo A, Hasselmann MH, Souza ER, Lozana JA, et al. The magnitude of intimate partner violence in Brazil: portraits from 15 capital cities and the Federal District. Cad Saúde Pública 2006; 22:425-37.

10. Fanslow J, Robinson E. Violence against women in New Zealand: prevalence and health consequences. N Z Med J 2004; 117:U1173.

11. Barber CF. Domestic violence against men. Nurs Stand 2008; 22:35-9.

12. Mechem CC, Shofer FS, Reinhard SS, Hornig S, Datner E. History of domestic violence among male patients presenting to an Urban Emergency Department. Acad Emerg Med 1999; 6:786-91.

13. Breiding MJ, Black MC, Ryan GW. Prevalence and risk factors of intimate partner violence in eighteen US states/territories, 2005. Am J Prev Med 2008; 34:112-8.

14. Centers for Disease Control and Prevention. Lifetime and annual incidence of intimate partner violence and resulting injuries - Georgia, 1995. MMWR Morb Mortal Wkly Rep 1998; 47:849-53.

15. Reid RJ, Bonomi AE, Rivara FP, Anderson ML, Fishman PA, Carrell DS, et al. Intimate partner violence among men prevalence, chronicity, and health effects. Am J Prev Med 2008; 34:478-85.

16. Centers for Disease Control and Prevention. Prevalence of intimate partner violence and injuries Washington, 1998. MMWR Morb Mortal Wkly Rep 2000; 49:589-92.
17. Centers for Disease Control and Prevention. Intimate partner violence among men and women South Carolina, 1998. MMWR Morb Mortal Wkly Rep 2000; 49:691-4.

18. Anacleto AJ, Njaine K, Longo GZ, Boing AF, Peres KG. Prevalência e fatores associados à violência entre parceiros íntimos: um estudo de base populacional em Lages, Santa Catarina, Brasil, 2007. Cad Saúde Pública 2009; 25:800-8.

19. Zaleski M, Pinsky I, Laranjeira R, Ramisetty-Mikler S, Caetano R. Violência entre parceiros íntimos e consumo de álcool. Rev Saúde Pública 2010; 44: 53-9.

20. Schraiber LB, d'Oliveira AFPL, França-Junior I, Diniz S, Portella AP, Ludermir AB, et al. Prevalência da violência contra a mulher por parceiro íntimo em regiões do Brasil. Rev Saúde Pública 2007; 41:797-807.

21. World Health Organization. WHO Multi-Country Study on Women's Health and Domestic Violence Against Women: summary report of initial results on prevalence, health outcomes and women's responses. Geneva: World Health Organization; 2005.

22. Schraiber LB, Latorre MRDO, França Jr. I, Segri NJ, D'Oliveira AFPL. Validade do instrumento WHO VAW STUDY para estimar violência de gênero contra a mulher. Rev Saúde Pública 2010; 44:658-66.

23. Boing AC, Peres KG, Boing AF, Hallal PC, Silva NN, Peres MA. Inquérito de saúde EpiFloripa: aspectos metodológicos e operacionais dos bastidores. Rev Bras Epidemiol 2014; 17:147-62.

24. World Health Organization. The Alcohol Use Disorder Identification Test: guideline for use in primary care. Geneva: World Health Organization; 2001.

25. Mendoza-Sassi RA, Beria JU. Prevalence of alcohol use disorders and associated factors: a population-based study using AUDIT in Southern Brazil. Addiction 2003; 98:799-804.

26. Barros MBA, Botega N, Dalgalarrondo P, MarínLeón L, Oliveira HB. Prevalence of alcohol abuse and associated factors in a population-based study. Rev Saúde Pública 2007; 41:502-9.

27. McHugh MC, Frieze IH. Intimate partner violence. Ann NY Acad Sci 2006; 1087:121-41.

28. Johnson MP, Ferraro KJ. Research on domestic violence in the 1990s: making distinctions. J Marriage Fam 2000; 62:948-63.

29. Archer J. Sex differences in physically aggressive acts between heterosexual partners: a meta-analytic review. Aggress Violent Behav 2002; 7:313-51.

30. Archer J. Sex differences in aggression between heterosexual partners: a meta-analytic review. Psychol Bull 2000; 126:651-80.

31. Straus M. Victims and aggressors in marital violence. Am Behav Sci 1980; 23:681-704.

32. d'Oliveira AFPL, Schraiber LB, França Jr. I, Ludermir AB, Portella AP, Diniz CS, et al. Fatores associados à violência por parceiro íntimo em mulheres brasileiras. Rev Saúde Pública 2009; 43:299-311. 
33. Moreno Martín F. La violencia en la pareja. Rev Panam Salud Pública 1999; 5:245-58.

34. Caetano R, Cunradi CB, Schafer J, Clark CL. Intimate partner violence and drinking patterns among white, black, and Hispanic couples in the US. J Subst Abuse 2000; 11:123-38.

35. Castro R, Riquer F. La investigación sobre violencia contra las mujeres en América Latina: entre el empirismo ciego y la teoría sin datos. Cad Saúde Pública 2003; 19:135-46.

36. Straus MA, Gelles RJ. Physical violence in American families: risk factors and adaptations to violence in 8,145 families. New Brunswick: Transaction Books; 1990.

37. Nagassar RP, Rawlins JM, Sampson NR, Zackerali J, Chankadyal K, Ramasir C, et al. The prevalence of domestic violence within different socio-economic classes in Central Trinidad. West Indian Med J 2010; 59:20-5.

38. Tuesca R, Borda M. Violencia física marital en Barranquilla (Colombia): prevalencia y factores de riesgo. Gac Sanit 2003; 17:302-8.

39. Vieira LJES, Pordeus AMJ, Ferreira RC, Moreira DP, Maia PB, Saviolli KC. Fatores de risco para violência contra a mulher no contexto doméstico e coletivo. Saúde Soc 2008; 17:113-25.
40. Departamento de Ações Programáticas e Estratégicas, Secretaria de Atenção à Saúde, Ministério da Saúde. Linha de cuidado para a atenção integral à saúde de crianças, adolescentes e suas famílias em situação de violência: orientações para gestores e profissionais de saúde. Brasília: Ministério da Saúde; 2010.

41. Kaysen D, Dillworth TM, Simpson T, Waldrop A, Larimer ME, Resick PA. Domestic violence and alcohol use: trauma-related symptoms and motives for drinking. Addict Behav 2007; 32:1272-83.

42. Leonard KE. Alcohol and intimate partner violence: when can we say that heavy drinking is a contributing cause of violence? Addiction 2005; 100:422-5.

43. Sullivan TP, Ashare RL, Jaquier V, Tennen H. Risk factors for alcohol-related problems among victims of partner violence. Subst Use Misuse 2012; 47:673-85.

44. Bennett L, O'Brien P. Effects of coordinated services for drug-abusing women who are victims of intimate partner violence. Violence Against Women 2007; 13:395-411.

Recebido em 05/Set/2013

Versão final reapresentada em 04/Jul/2014

Aprovado em 10/Out/2014 